Copyright (C) 2006 IEEE. Reprinted from

IEEE Photonics Technology Letters, 2006; 18 (1-4):394-396

This material is posted here with permission of the IEEE. Such permission of the IEEE does not in any way imply IEEE endorsement of any of the University of Adelaide's products or services. Internal or personal use of this material is permitted. However, permission to reprint/republish this material for advertising or promotional purposes or for creating new collective works for resale or redistribution must be obtained from the IEEE by writing to pubs-permissions@ieee.org.

By choosing to view this document, you agree to all provisions of the copyright laws protecting it. 


\title{
Signature of Structure Failure Using Asymmetric and Broadening Factors of Brillouin Spectrum
}

\author{
Fabien Ravet, Student Member, IEEE, Xiaoyi Bao, Senior Member, IEEE, Togay Ozbakkaloglu, and Murat Saatcioglu
}

\begin{abstract}
We introduce a novel data analysis approach based on the extraction of peak strain, asymmetric and broadening factors of the Brillouin spectrum measured with the distributed Brillouin sensor (DBS). Such an approach provides simultaneously global and local strains, describing the status of the structure, at the contrary of average strain measurements. These results are confirmed by a trial on a composite column subjected to vertical and bending loads. This demonstrates that the DBS is a powerful tool to give the signature of the structure failure and then to identify early problems in structures that none of existing point sensors can detect.
\end{abstract}

Index Terms-Brillouin spectrum, distributed Brillouin sensor (DBS), form factors, strain sensor, structural health monitoring (SHM).

\section{INTRODUCTION}

O TRUCTURAL health monitoring (SHM) is used to identify early signs of potential problems, allowing prevention of disasters and then the repair of these damages. SHM is also implemented to improve the construction processes and to produce new building materials, so that it saves millions to billions of dollars over a structure's lifetime. Currently, techniques used for SHM are punctual devices giving only partial information on the stresses and not on the status of the structure. Their localized nature not only fails to give global information on the structure health, they are also incapable of detecting local defects when location is not known in advance.

These difficulties can be overcome by distributed strain measurements in real time as do distributed Brillouin sensor (DBS) systems [1]. Recent studies [2], [3] have been conducted to monitor strain of composite and concrete beams. Strain measurements are based on the extraction of the average value of the Brillouin spectrum, which is the most commonly used signal processing technique [1]-[3]. If the spectrum has a symmetric shape under the condition of low pump and probe power, this means the asymmetric feature of the Brillouin spectrum is induced only by the stress condition. Then average strain reflects the overall structure condition. Global and local strains almost coincide, so the structure does not suffer deformations, cracks, or materials debonding. The coincidence of global and local strains is the condition for the concrete beam to be in elastic regime as it happens in laboratory testing. The beam follows the loading condition linearly and reversibly [2].

Manuscript received July 21, 2005; revised November 3, 2005. This work was supported by Intelligent Sensing for Innovative Structures (ISIS) Canada and by the Natural Science and Engineering Research Council of Canada.

F. Ravet and X. Bao are with the Department of Physics, University of Ottawa, Ottawa, ON K1N 6N5, Canada (e-mail: fabien.ravet@ieee.org).

T. Ozbakkaloglu and M. Saatcioglu are with the Civil Engineering Department, University of Ottawa, Ottawa, ON K1N 6N5, Canada.

Digital Object Identifier 10.1109/LPT.2005.862366
However, when civil engineering structures are subjected to substantial loads, induced by earthquakes or severe weather conditions for example, they start to fail, so debonding and deformation are developing. The Brillouin profile becomes locally asymmetric and the peak is broadened (assuming the pulsewidth is fixed) with the full-width at half-maximum (FWHM) much bigger than the Brillouin spectrum linewidth $\left(\Delta \nu_{B}\right)$ of the unstressed structure $\left(\mathrm{FWHM}>\Delta \nu_{B} \approx 40 \mathrm{MHz}\right.$ for SMF-28). Brillouin peak frequency does not match the mass-weight center of the spectrum and average strain value. Peak frequency measurement obtained by the curve fitting technique gives only global strain information. Moreover, the average strain does not represent the local strain associated with cracks and deformation of the structure as well as fiber reinforced polymer (FRP)/concrete debonding. We find that the early sign of structural failures is related to asymmetry and broadening of the Brillouin spectrum.

Our signal processing approach requires both high spatial and frequency resolutions. In commercial DBS systems, this requirement is impossible to achieve due to the pulse-induced spectral broadening. In our sensor, that effect is mitigated by taking advantage of the existence of a dc part to the pulse which contributes to coherent probe(pulse)-pump(continuous wave) interaction [5].

\section{Proposed Data AnAlysis Methodology}

We propose a novel signal processing approach to extract both global and local strain informations. We keep the Brillouin peak measurement as the global strain tag but we introduce, for the first time in distributed sensing field, two form factors, asymmetric $\left(F_{A}\right)$ and broadening $\left(F_{B}\right)$ factors, that are the signatures of local degradations such as cracks, deformation, and debonding. The form factors are defined as $F_{A}=\Delta \nu_{R} / \Delta \nu_{L}$ and $F_{B}=\Delta \nu_{\mathrm{Bs}} / \Delta \nu_{B}$, where $\Delta \nu_{R}\left(\Delta \nu_{L}\right)$ and $\Delta \nu_{\mathrm{Bs}}$ are, respectively, the right (left) half-width at half-maximum and the FWHM of the broadened Brillouin loss spectrum. Brillouin loss spectra measured at every location are analyzed to extract these three parameters as illustrated in Fig. 1.

The first parameter $\left(\nu_{\mathrm{pk}}\right)$ is the frequency shift that gives us global information on the longest strained section observed within the pulsewidth $(w)$ at a given position in the fiber $(z)$. The second parameter $F_{A}$ indicates the presence of higher but short strain components. Finally, $F_{B}$ describes the broadening of the Brillouin loss spectrum induced by nonuniform strain distribution. We discuss various strain regimes associated with the form factors value. Fig. 2(a) and (b) shows various cases with the same $\nu_{\mathrm{pk}}$ but different strain distributions. These spectra are the results of simulations [4] for given pump and probe conditions $\left(P_{\text {pump }}=10 \mathrm{~mW}, P_{\text {probe }}=5 \mathrm{~mW}\right)$ at the same 


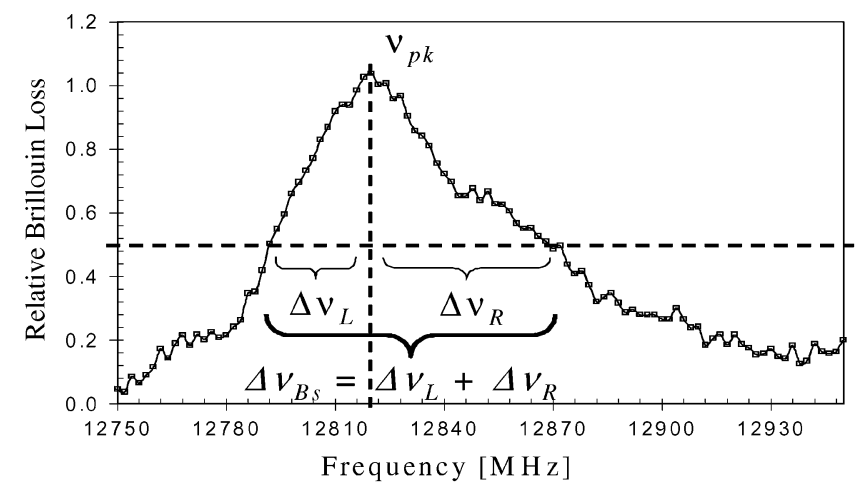

Fig. 1. Definition of the width parameters on an experimental Brillouin loss spectrum of a strained section of a single-mode optical fiber.
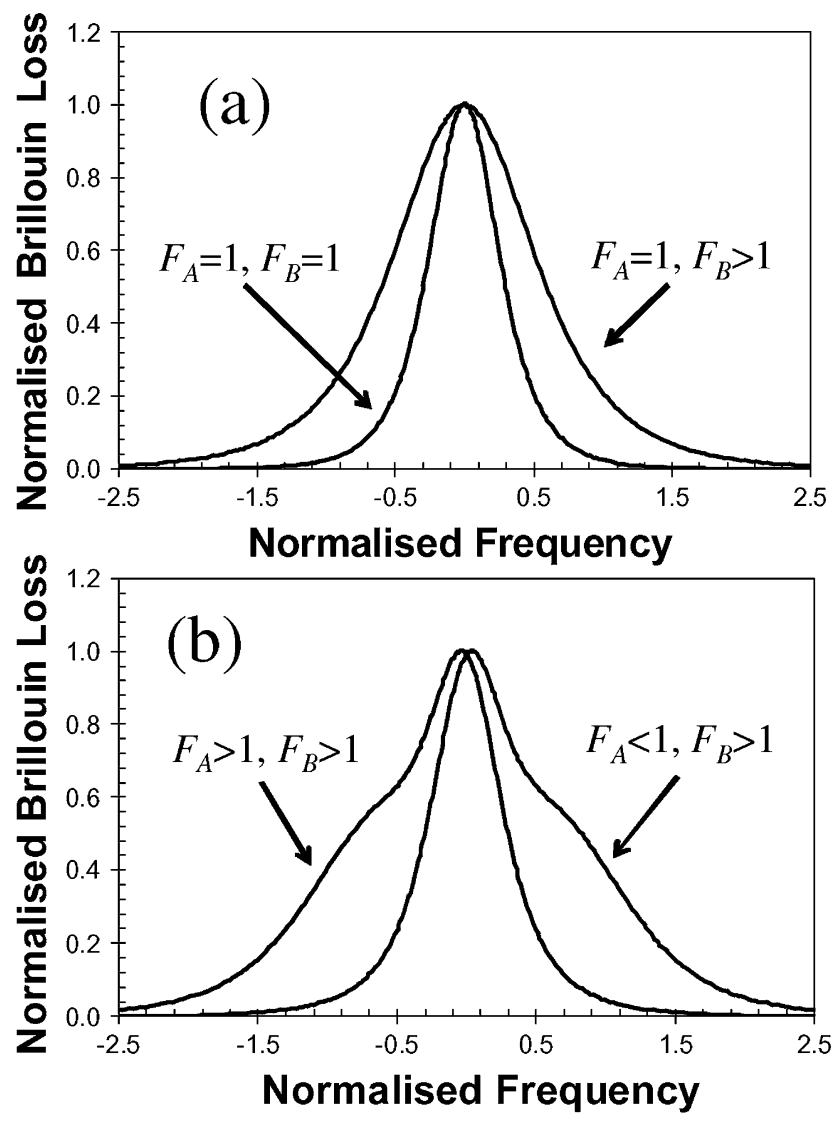

Fig. 2. Results of numerical simulations [4] for various strain profiles included within $w$ : (a) uniform strain $\left(F_{A}=1, F_{B}=1\right)$, linear strain $\left(F_{A}=1, F_{B}>1\right)$; (b) nonlinear strain with short components larger than main strain contribution $\left(F_{A}>1, F_{B}>1\right)$, nonlinear strain with short components smaller than main strain contribution $\left(F_{A}<1, F_{B}>1\right)$.

location in the fiber (fiber length is $40 \mathrm{~m}$ and $z=20 \mathrm{~m}$ ) and identical pulsewidth $(w=2 \mathrm{~m})$. When $F_{A}=1$ and $F_{B}=1$, the strain distribution is uniform. The spectrum is simply shifted and a peak-finding approach is enough to characterize the status of the structure [Fig. 2(a)]. If the spectrum is still symmetric $\left(F_{A}=1\right)$ but $F_{B}>1$, then the distribution is nonuniform [6]. The peak-finding technique describes the global behavior of the structure but it fails to detect the presence of strain over section shorter than pulsewidth. The strain distribution becomes asymmetric when $F_{A} \neq 1$ as in Fig. 2(b). For $F_{A}>1$, the strain distribution is nonuniform corresponding to large (a)

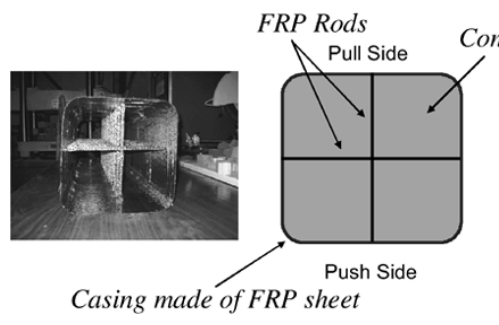

Fig. 3. (a) Column cross section without concrete showing the rods laid within the FRP sheets casing. (b) Column cross section schematic. (c) View of the bottom of the column with the four first optical fiber layers.

local strain components while the global strain $\left(\nu_{\mathrm{pk}}\right)$ is low. It indicates that small defects start to build up in the structure. When $F_{A}<1$, global strain becomes the highest component. It means that the structure is in jeopardy because the local strain becomes the dominating contribution. Apparently, the use of $F_{A}$ and $F_{B}$ introduces two advantages: 1) Even if the global strain is the same (same $\nu_{\mathrm{pk}}$ ), they show distinct structural status; 2) it provides a complete picture compared to average strain detection or multiple peak analysis.

\section{BRILLOUIN SENSOR CHARACTERISTICS}

Common DBSs are limited in frequency resolution when pulsewidth is smaller than the acoustic phonon lifetime due to pulse induced spectral broadening. At the contrary, we developed and designed our sensor to take advantage of the influence of the electrooptic modulator finite extinction ratio (ER) that enhances spatial resolution [5]. The dc component of the pulse interacts with the pump giving two contributions to the loss spectrum: 1) the interaction of the component present in the dc part of the probe with the pump gives a spectrum characteristic of the whole fiber; 2) the interaction of the pulse part of the probe with the pump gives a spectrum characteristic of local stress. Eventually, the Brillouin loss signal is enhanced and narrower than the signal that would be produced by the pulse-pump interaction only (infinite ER case). In our experiment, we used an ER of $15 \mathrm{~dB}$ which keeps the spectrum FWHM within a few percent of the Brillouin gain natural linewidth under unstrained or uniform strain conditions. A nonuniform strain spectrum measured with a 1.7-ns pulse, which is equivalent to a spatial resolution of $17 \mathrm{~cm}$, is presented in Fig. 1. Spectra are taken every $5 \mathrm{~cm}$ (which is the sampling interval of sensor digitizer) allowing us to achieve centimeter-size crack detection [4], [5]. Our sensor operates at $1309 \mathrm{~nm}$. The sensing medium is a single-mode optical fiber.

\section{EXPERIMENT DESCRIPTION}

We applied our new spectrum analysis scheme to the monitoring of a concrete/FRP structure subjected to extreme stresses. Due to their composite nature, the structures suffer very distinct damages, which eventually lead to collapse. These degradations are FRP/concrete debonding, crack as well as shape deformation. The concrete column using FRP rods as reinforcing bars is wrapped in FRP sheets [Fig. 3(a) and (b)]. An optical fiber is laid along the column. The fiber is glued horizontally at ten distinct cross sections of the column (from bottom, Layer 1, to the top, Layer 10). Each section is $1 \mathrm{~m}$ long and separated by 


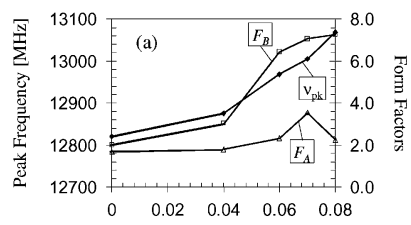

drift

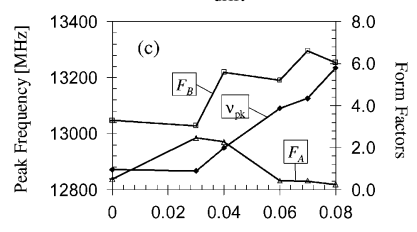

drift

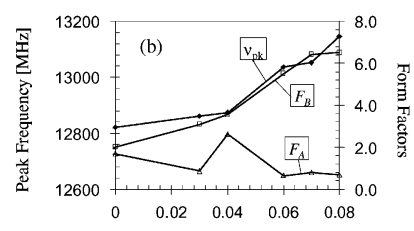

drift

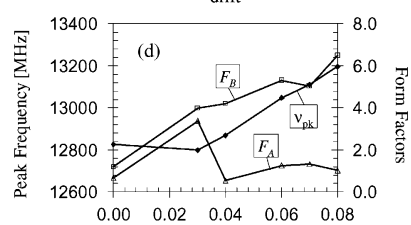

drift
Fig. 4. Peak frequency (left $y$-axis) and form factors (right $y$-axis) as a function of column drift: (a) push side, Layer 4, median point, push condition; (b) push side, Layer 4, median point, pull condition; (c) push side, Layer 2, median point, push condition; (d) pull side, Layer 4 , median point, pull condition.

$1 \mathrm{~m}$ of loose fiber. An optical pulse is launched at the end of the fiber located at the top of the column (Layer 10). An axial load of $1880 \mathrm{kN}$ is applied on the column, while it is pushed and pulled, to represent the real load of the building. Push and pull refers to the location of the observer [Fig. 3(b)]. Pull corresponds to the column side facing the observer. The opposite face is associated with the push action. The push and pull amplitudes are measured by the drift, varied from 3 to $8 \%$. The drift parameter is defined as the ratio of the lateral displacement $(\delta l)$ to the height of the column $(h)$. The drift is gradually increased by $1 \%$ step until the column collapses which occurred at $>8 \%$ drift. Measurements with the Brillouin sensor are taken at each drift step.

\section{RESUlts ANALYSIS}

From each Brillouin measurement, we extracted $\nu_{\mathrm{pk}}$ and calculated $F_{A}$ and $F_{B}$. We then drew $\nu_{\mathrm{pk}}$ and the two form factors as a function of the drift amplitude for both push and pull condition. We concentrated our analysis on two fiber sections located on the median of Layers 2 and 4 of the push side [Fig. 3(c)]. We also analyzed the median point of Layer 4 of pull (i.e., symmetric of Layer 3 push side).

The graphs of Fig. 4 show that $\nu_{\mathrm{pk}}$ and $F_{B}$ experience monotonic increase with a bend in the curve when $F_{A} \approx 2$ and $F_{B} \approx 3$. At larger drifts, $F_{B}$ tends to saturate [Fig. 4(a) and (b)] or to fluctuate [Fig. 4(c) and (d)]. $F_{A}$ increases above two (it can reach a maximum of four) and then drops below one and becomes steady. The region of smaller $\nu_{\mathrm{pk}}$ slope corresponds to elastic regime of the column without deformation. The increase of $F_{A}$ and $F_{B}$ values are associated with the appearance of local stresses, contributing to the crushing and deformations of the concrete. The bend in $\nu_{\mathrm{pk}}$ is followed by a sudden slope rise, when $F_{A} \approx 2$ and $F_{B} \approx 3$, which is due to the complete concrete collapse as observed in the experiment. FRP and concrete are fully debonded, the structure is maintained by the FRP only, leaving it free to follow the load changes. Ultimately, $F_{B}$ saturates and/or fluctuates when the drift becomes large and $F_{A} \leq 1$. Large strain is the dominant contribution. The column safety is then threatened. The FRP, being the only element sup-
TABLE I

SIGNATURE OF STRUCTURE FAILURE WITH FORM FACTORS

\begin{tabular}{|c|c|c|c|c|}
\hline $\begin{array}{c}\text { Strain } \\
\text { Observation }\end{array}$ & $F_{A}$ & $F_{B}$ & $\begin{array}{c}v_{p k} \\
\text { Slope }\end{array}$ & Structure Status \\
\hline Uniform & $<2$ & $\begin{array}{l}<3 \\
\text { Small } \\
\text { slope }\end{array}$ & $\begin{array}{l}\text { Constant, } \\
\text { small }\end{array}$ & $\begin{array}{l}\text { Elastic regime, } \\
\text { good shape }\end{array}$ \\
\hline $\begin{array}{l}\text { Non-uniform, low } \\
\text { strain dominates }\end{array}$ & $\begin{array}{c}>2 \\
\text { Peak value }\end{array}$ & $\begin{array}{l}>3 \\
\text { Large } \\
\text { slope }\end{array}$ & Increase & $\begin{array}{l}\text { Deformation, local } \\
\text { cracks, local de- } \\
\text { bonding }\end{array}$ \\
\hline $\begin{array}{l}\text { Non-uniform, large } \\
\text { strain dominates }\end{array}$ & $\begin{array}{l}<1 \\
\text { Stationary }\end{array}$ & $\begin{array}{c}>>3 \\
\text { Constant } \\
\text { and large } \\
\text { slope }\end{array}$ & $\begin{array}{l}\text { Constant, } \\
\text { Large }\end{array}$ & $\begin{array}{c}\text { Full FRP/concrete } \\
\text { de-bonding }\end{array}$ \\
\hline $\begin{array}{l}\text { Reduced non- } \\
\text { uniformity, large } \\
\text { strain dominates }\end{array}$ & $\begin{array}{c}<1 \\
\text { Stationary }\end{array}$ & $\begin{array}{c}\gg 3 \\
\text { Stationary }\end{array}$ & $\begin{array}{l}\text { Constant, } \\
\text { Large }\end{array}$ & FRP cracks \\
\hline
\end{tabular}

porting the structure, starts to rupture locally. These ruptures release locally the tension inducing a local strain reduction and then mitigating the spectrum broadening.

Table I summarizes the relationship of the structure behavior with the three parameters variation. Monitoring the changes of these values can predict the early sign of collapse. The conclusions drawn in Table I are valid for 1.7-ns pulses but should not be affected by pulsewidth of the same order of magnitude, which is the best spatial resolution that can be currently achieved.

\section{CONCLUSION}

A new signal processing approach is introduced based on the shape analysis of the Brillouin spectrum. Asymmetric and broadening factors of the Brillouin spectrum are associated with the degradation of the column. These parameters are a clear signature of structure failures. It strengthens the DBS position as a serious candidate for SHM applications.

\section{REFERENCES}

[1] T. Horiguchi, K. Shimizu, T. Kurashima, M. Tateda, and Y. Koyamada, "Development of a distributed sensing technique using brillouin scattering," J. Lightw. Technol., vol. 13, no. 7, pp. 1296-1302, Jul. 1995.

[2] X. Zeng, X. Bao, C. Y. Chhoa, T. W. Bremner, A. W. Brown, M. D. DeMerchant, G. Ferrier, A. L. Kalamkarov, and A. V. Georgiades, "Strain measurement in a concrete beam by use of the Brillouin-scattering-based distributed fiber sensor with single-mode fibers embedded in glass fiber reinforced polymer rods and bonded to steel reinforcing bars," Appl. Opt., vol. 41, pp. 5105-5114, 2002.

[3] H. Murayama, K. Kageyama, H. Naruse, A. Shimada, and K. Uzawa, "Application of fiber-optic distributed sensors to health monitoring for full-scale composite structures," J. Intell. Mat. Syst. Struct., vol. 14, pp. 3-13, 2003.

[4] F. Ravet, X. Bao, Q. Yu, and L. Chen, "Criterion for subpulse-length resolution and minimum frequency shift in distributed Brillouin sensors," IEEE Photon. Technol. Lett., vol. 17, no. 7, pp. 1504-1506, Jul. 2005.

[5] L. Zou, X. Bao, Y. Wan, and L. Chen, "Coherent probe-pump-based brillouin sensor for centimeter-crack detection," Opt. Lett., vol. 15, pp. 370-372, 2005.

[6] H. Naruse, M. Tateda, H. Ohno, and A. Shimada, "Dependence of the brillouin gain spectrum on linear strain distribution or optical time-domain reflectometer-type strain sensors," Appl. Opt., vol. 41, pp. 7212-7217, 2002. 